\title{
Delaying the Decay of a Superposition of Resonance States
}

\author{
A. García-Vela* \\ Instituto de Física Fundamental, Consejo Superior de Investigaciones Científicas, \\ Serrano 123, 28006 Madrid, Spain
}

\begin{abstract}
A weak-field coherent control scheme is applied in order to enhance the decay lifetime of a superposition of overlapping resonance states. The scheme uses a pump laser field consisting of two pulses delayed in time, each of them exciting a different energy at which several resonances of the $\mathrm{Ne}_{-} \mathrm{Br}_{2}(B)$ complex overlap. Simultaneous excitation of these two energies induces interference between the overlapping resonances, which causes an enhancement of the lifetime of the superposition created. By varying the delay time between the pulses, the mechanism of resonance interference can be controlled and optimized to achieve a maximum lifetime enhancement. The optimal delay time between pulses leading to maximum superposition lifetime can be quantitatively predicted with a simple law. The effect of the interference mechanism on the lifetime enhancement is investigated. It is found that interference induces a transfer of amplitude between the different resonances back and forth, which delays significantly the natural resonance decay, increasing the global lifetime of the superposition. Due to the simplicity of the control scheme, a wide applicability is envisioned.
\end{abstract}

E-mail: garciavela@iff.csic.es 


\section{Introduction}

The study of decoherence in molecular systems has received an increasing research interest in the last years. ${ }^{1,2}$ Molecular decoherence, also called "molecular dephasing", ${ }^{3}$ is a process in which the phase coherence of a molecular superposition state prepared by an external field is lost with time due to the coupling of the molecule to an environment. Actually, decoherence involves a loss of quantum information of the molecular system (the loss of coherence of the molecular superposition) when the environment is neglected. Thus decoherence has important implications in the areas of quantum computation and quantum information processing, ${ }^{4}$ and the development of techniques to avoid or delay the effects of decoherence is of great interest. In this sense, a variety of schemes to control decoherence has been proposed. ${ }^{5-11}$

While molecular decoherence is traditionally associated with open systems composed of a molecular chromophore coupled to an external environment, there exists a close analogue to decoherence in isolated molecules, which is the process of intramolecular vibrational redistribution (IVR). In the IVR process, a superposition of molecular states prepared by a laser field loses coherence along time due to the coupling of the different states of the superposition to other degenerate vibrational or vibronic states of the molecule. The loss of coherence is caused by the different coupling of each vibrational state forming the molecular superposition to the corresponding degenerate molecular states, what produces a different, nonuniform decay of the amplitude excited in the different states of the superposition. IVR is usually the origin of many radiationless relaxation processes in molecular systems, which prevent to keep a molecule in an excited superposition state for a long time. Thus, similarly as with decoherence, it is desirable to avoid or delay as much as possible the effects of IVR, and this challenge has been actively pursued.

A successful coherent control scheme of the IVR of a molecular superposition state composed of overlapping resonances has been suggested. ${ }^{12}$ The performance of the control scheme was demonstrated under weak-field (one photon) conditions 
for a model of the vibrational dynamics of the OCS molecule. The scheme exploits the effect of quantum interference between the overlapping resonances of the superposition that can be induced by a properly chosen laser field. By using optimal control theory (OCT) techniques, ${ }^{13}$ the relative amplitudes of the different overlapping resonances in the superposition prepared were optimized such that interference between them led to a significant delay or an enhancement of the effects of IVR. ${ }^{12}$ In a practical experiment, the optimization of the resonance amplitudes in the molecular superposition can be achieved by applying a laser field properly taylored by means of pulse shaping techniques. This type of coherent control of IVR in molecular superpositions of overlapping resonances was also applied to control radiationless transitions like internal conversion in pyrazine. ${ }^{14,15}$

In the last years, new weak field coherent control schemes based on the quantum interference effect between overlapping resonances have been suggested. ${ }^{16-21}$ The main goal of those schemes was not to control the IVR effects of the whole superposition of resonances created, as done in the previous works of refs $12,14,15$, but to control instead the behavior of a single specific resonance within the superposition. In other words, the schemes pursued control of the two main properties of a given resonance in the superposition, namely the resonance lifetime ${ }^{16-18,21}$ and the fragment state distribution produced upon resonance decay. ${ }^{19-21}$ The schemes typically applied a laser field consisting of two Gaussian pulses with a time delay between them. ${ }^{17,18,20,21}$ Resonance lifetime control is achieved when the two pulses overlap in time to some extent. Then, by varying the delay time between the pulses, the relative amplitudes of the different overlapping resonances in the superposition can be modified, allowing for control of the intensity of the interference between them. In this way interference between resonances can be optimized in order to maximize the lifetime of a given resonance. Thus, optimization of the laser field applied is carried out by fitting properly the delay time between the two pulses, and eventually their realtive amplitudes. ${ }^{17}$ In this sense this control scheme is simpler than that of ref 12 , because it does not require a pulse shaper to optimize the field. 
While the original aim of the control scheme was to enhance the lifetime of a given single resonance out of the superposition prepared, it is clear that the quantum interference induced between the different overlapping resonances will also affect the survival (and therefore its IVR decay behavior) of the whole superposition. This aspect of the control scheme has not been investigated so far. Thus the main goal of the present work is to study the conditions and mechanisms by means of which application of the above control scheme can maximize the survival of a superposition of overlapping resonances. The current control goal is therefore similar to that of ref. 12, namely to modify the decay lifetime of a superposition of overlapping resonances by inducing interference between them, but applying a simpler control strategy in the present case. As in previous works, vibrational predissociation of the $\mathrm{Ne}-\mathrm{Br}_{2}(B)$ complex has been chosen as the molecular process subject of this study, because this is a system that features different types of resonances, among them overlapping resonances. Resonances are common to several types of weakly-bound complexes. $^{22,23}$

The paper is organized as follows. In section II the methodology used is briefly described. The results are presented and discussed in section III. Finally, some conclusions are given in section IV.

\section{Theoretical Methodology}

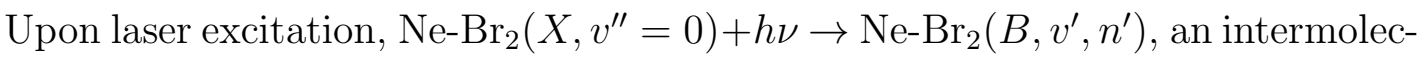

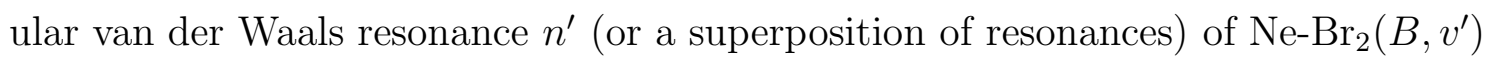
is populated. The labels $v^{\prime \prime}$ and $v^{\prime}$ denote the vibrational states of $\mathrm{Br}_{2}$ in the $X$ and $B$ electronic states, respectively, while the $n^{\prime}$ index labels the energy position of the resonance, with $n^{\prime}=0$ corresponding to the ground one. Then the resonance excited decays to the fragmentation continuum through vibrational predissociation, $\mathrm{Ne}-\mathrm{Br}_{2}\left(B, v^{\prime}, n^{\prime}\right) \rightarrow \mathrm{Ne}+\mathrm{Br}_{2}\left(B, v_{f}<v^{\prime}\right)$. This process has been studied in detail both experimentally ${ }^{24,25}$ and theoretically ${ }^{26,27}$.

The Ne- $\mathrm{Br}_{2}\left(B, v^{\prime}, n^{\prime}\right)$ excitation with a laser field and the subsequent predisso- 
ciation was simulated with a full three-dimensional wave packet method (assuming $J=0$ ) described in detail elsewhere ${ }^{16,26}$. In order to asses the quality of the model applied, it is noted that the lifetime calculated with the present theoretical model for the decay of the $\mathrm{Ne}_{-} \mathrm{Br}_{2}\left(B, v^{\prime}=16\right)$ ground intermolecular resonance was found to be $69 \mathrm{ps},{ }^{28}$ while the corresponding lifetime estimated experimentally is $68 \pm 3 \mathrm{ps}^{25}$ This good agreement implies that both the three-dimensional wave packet method and the potential surfaces used in the present simulations are realistic enough in order to describe this resonance decay process.

The present simulations focus on excitation of the $\mathrm{Ne}_{-\mathrm{Br}_{2}}\left(B, v^{\prime}=35, n^{\prime}=0\right)$ ground intermolecular resonance. The excitation spectrum associated with such resonance is displayed in Figure 1, and has been previously investigated. ${ }^{29}$ The main peak located at the energy $-56.34 \mathrm{~cm}^{-1}$ (relative to the $\mathrm{Ne}+\operatorname{Br}_{2}\left(B, v^{\prime}=\right.$ $\left.35, j^{\prime}=0\right)$ dissociation threshold $)$ is associated with the $\mathrm{Ne}-\mathrm{Br}_{2}\left(B, v^{\prime}=35\right)$ ground resonance, labeled as $\left(v^{\prime}, g r\right)$ in the figure. This resonance lies below the $\mathrm{Ne}+$ $\operatorname{Br}_{2}\left(B, v^{\prime}-1=34, j^{\prime}=0\right)$ dissociation threshold, and therefore it is embedded in the spectrum of intermolecular resonances of the $v^{\prime}-1=34$ vibrational manifold, which is responsible of the remaining features of the spectrum of Figure 1. Resonances in the range $n=5-13$ that overlap between them and also with the $v^{\prime}$ ground resonance are identified in Figure 1.

In the simulations reported here two energies of the spectrum of Figure 1 are excited simultaneously by a pump laser field consisting of the combination of two Gaussian pulses, each pulse exciting one of the two energies. The two energies excited are that associated with the peak of the $n^{\prime}=0$ ground resonance of the $v^{\prime}=35$ manifold, $E_{n^{\prime}=0}$, and that associated with the peak of the $n=9$ intermolecular resonance of the $v^{\prime}-1$ manifold, $E_{n=9}$, (located at $-57.75 \mathrm{~cm}^{-1}$ ), which overlaps with the $v^{\prime}$ ground resonance. Although it was previously determined ${ }^{29}$ that the main contribution to these two peaks comes from the $n^{\prime}=0$ and $n=9$ resonances, respectively, it is noted that in a strong overlapping regime like that of Figure 1, actually several resonances overlap at each of both energies. ${ }^{30}$ The implication is that each 
Gaussian pulse of the field will populate all the resonances overlapping at the corresponding excitation energy. When the two energies are populated simultaneously, interference between those overlapping resonances is induced.

The pump laser field applied can be expressed as

$$
\mathcal{E}_{\text {pump }}(t)=A_{1} e^{-\left(t-t_{1}\right)^{2} / 2 \sigma^{2}} \cos \left[\omega_{1}\left(t-t_{1}\right)+\phi_{1}\right]+A_{2} e^{-\left(t-t_{2}\right)^{2} / 2 \sigma^{2}} \cos \left[\omega_{2}\left(t-t_{2}\right)+\phi_{2}\right],
$$

where $\omega_{1}$ and $\omega_{2}$ are the photon frequencies required to excite the energy of the $n^{\prime}=0$ and $n=9$ resonance peaks, respectively, and $t_{1}$ and $t_{2}$ are the time centers of the two pulses. In practice $t_{1}$ is always fixed at the value $t_{1}=0$, and $t_{2}$ is varied. Thus the delay time between the pulses becomes $\Delta t=t_{2}-t_{1}=t_{2}$. A temporal width (related to $\sigma$ ) of FWHM=200 ps is used for the two Gaussian pulses, which has associated a spectral width of $0.15 \mathrm{~cm}^{-1}$, which implies that both pulses are spectrally very narrow and do not overlap in energy. For simplicity it was assumed that $\phi_{1}=\phi_{2}=0$. The amplitudes of the pulses are also assumed to be equal, $A_{1}=A_{2}=1.0 \times 10^{-6} E_{h} /\left(e a_{0}\right)$, which corresponds to a maximum pulse intensity of about $3.5 \times 10^{4} \mathrm{~W} / \mathrm{cm}^{2}$, within the weak-field regime.

When the laser field $\mathcal{E}_{\text {pump }}(t)$ is applied to excite the energies $E_{n^{\prime}=0}$ (excited by the pulse centered at $t_{1}$ ) and $E_{n=9}$ (excited by the pulse centered at $t_{2}$ ), a wave packet is created,

$$
\Phi(t)=\xi_{E_{n^{\prime}=0}}(t)+\xi_{E_{n=9}}(t)
$$

with $\xi_{E_{n^{\prime}=0}}(t)$ and $\xi_{E_{n=9}}(t)$ being the amplitudes excited around $E_{n^{\prime}=0}$ and $E_{n=9}$ by the two pulses of $\mathcal{E}_{\text {pump }}(t)$. As mentioned above, the single wave packets $\xi_{E_{n^{\prime}=0}}(t)$ and $\xi_{E_{n=9}}(t)$ have a very narrow spectral width around the energies $E_{n^{\prime}=0}$ and $E_{n=9}$, respectively, although each of them populate several resonances that overlap at each energy. Due to this narrow bandwidth of $\xi_{E_{n^{\prime}=0}}(t)$ and $\xi_{E_{n=9}}(t)$, interference between the overlapping resonances populated by each wave packet is negligible when they are created separately, in a nonsimultaneous way. Indeed, these narrow-bandwith wave packets contain essentially a single-energy component in practice, which cannot interfere with itself. 
However, when the two wave packets $\xi_{E_{n^{\prime}=0}}(t)$ and $\xi_{E_{n=9}}(t)$ are created simultaneously by $\mathcal{E}_{\text {pump }}(t)$, interference between the resonances overlapping at both $E_{n^{\prime}=0}$ and $E_{n=9}$ takes place. Thus, the whole wave packet $\Phi(t)$ created by $\mathcal{E}_{\text {pump }}(t)$ consists of a superposition of overlapping resonances excited at two different energies, $E_{n^{\prime}=0}$ and $E_{n=9}$, that will interfere. Since the resonances decay to the continuum after excitation by the laser field, the relative amplitudes of the different overlapping resonances in the superposition prepared can be varied by changing the delay time $\Delta t$ between the two pulses of $\mathcal{E}_{\text {pump }}(t)$. In this way the mechanism of interference between resonances can be controlled and optimized in order to maximize the survival of the superposition $\Phi(t)$ prepared.

The survival probability of $\Phi(t)$ along time can be expressed as

$$
I(t)=|<\Phi(t)| \Phi(t)>\mid .
$$

Now, by varying the delay time $\Delta t$ between the pulses of $\mathcal{E}_{\text {pump }}(t)$, superpositions of the resonances overlapping at the $E_{n^{\prime}=0}$ and $E_{n=9}$ energies are prepared with different relative amplitudes. The time evolution of each superposition $\Phi(t)$ is computed, and the corresponding survival probability $I(t)$ is calculated. Then, in order to extract a lifetime associated with each $I(t)$ curve, they are fitted to the function ${ }^{24,26}$

$$
I_{f i t}(t)=A \int_{-\infty}^{t} C C\left(t^{\prime}\right)\left[\exp \left(-\left(t-t^{\prime}\right) / \tau\right)\right] d t^{\prime},
$$

being $C C\left(t^{\prime}\right)$ the laser cross-correlation curve $\left(\mathcal{E}_{\text {pump }}^{2}\left(t^{\prime}\right)\right)$ and $A$ an amplitude scaling parameter related to the overlap factor between the initial and the final states coupled by the laser field. The above expression reflects the convolution of the two processes that contribute to generate the transient signal $I(t)$, namely the pumping of amplitude to the superposition of resonances by the laser field and the exponential resonance decay of this amplitude along time, and it is widely used. ${ }^{24}$ In this way a lifetime $\tau$ is obtained for each superposition created for different values of $\Delta t$.

\section{Results and Discussion}

As mentioned above, when a single energy (either $E_{n^{\prime}=0}$ or $E_{n=9}$ ) is excited by one 
of the two pulses of $\mathcal{E}_{\text {pump }}(t)$, a superposition of the overlapping resonances reflected in the spectrum of Figure 1 is created with different contributions. By proyecting out the single-pulse wave packet prepared in this way, $\xi_{E_{n^{\prime}=0}}(t)$ or $\xi_{E_{n=9}}(t)$, onto the different resonance wave functions, $\psi_{n^{\prime}=0}$ and $\psi_{n}$, the corresponding contributions $I_{k}(t)=\left|<\psi_{k}(t)\right| \xi_{E_{l}}(t)>\left.\right|^{2}\left(k=n^{\prime}, n\right.$ and $l$ standing for $n^{\prime}=0$ and $\left.n=9\right)$ to the survival probability $I(t)$ of the superposition can be obtained. In the case of $E_{n^{\prime}=0}$, the main contributions to the resonance superposition come from the $n^{\prime}=0$, $n=8,9$, and 11 resonances (with $n=9$ and 11 being the dominant ones among the $v^{\prime}-1=34$ resonances). In addition, the $n=7$ and 10 resonances have a smaller but significant contribution, and the remaining $n=5,6,12$, and 13 resonances (which are farther away from $E_{n^{\prime}=0}$ ) contribute in a rather marginal or negligible way. For the superposition created when exciting $E_{n=9}$, the situation is rather similar, with the main contributions corresponding to $n=9, n^{\prime}=0$, and $n=8$. Now $n=7$ and 11 have a small but appreciable contribution, and the remaining resonances contribute negligibly. Thus, for the two energies the corresponding superposition prepared mostly consists of the $3-4$ resonances that overlap more strongly at those energies. As already discussed, the resonances of these narrow bandwidth singlepulse superpositions cannot interfere appreciably.

The time evolution of the survival probability of the two single-pulse superpositions created separately at the $E_{n^{\prime}=0}$ and $E_{n=9}$ energies has been computed. By applying eq 4 a lifetime is estimated for each superposition. The resulting superposition lifetime is expected to be an average of the lifetimes of the different resonances weighted by their contribution to the specific superposition. The lifetimes associated with the $E_{n^{\prime}=0}$ and $E_{n=9}$ superpositions are found to be $\tau \sim 4$ and $\sim 15 \mathrm{ps}$, respectively. Not surprisingly, these lifetimes are quite similar to the lifetimes obtained for the single-pulse survival probabilities $I_{n^{\prime}=0}(t)=\left|<\psi_{n^{\prime}=0}(t)\right| \xi_{E_{n^{\prime}=0}}(t)>\left.\right|^{2}$ and $I_{n=9}(t)=\left|<\psi_{n=9}(t)\right| \xi_{E_{n=9}}(t)>\left.\right|^{2}$ of the $n^{\prime}=0$ and $n=9$ resonances that contribute dominantly at each energy, which are $\tau=3.8$ and $13.0 \mathrm{ps}$, respectively. ${ }^{18}$

Now, in order to induce interference between the overlapping resonances, the two- 
pulse field $\mathcal{E}_{\text {pump }}(t)$ of eq 1 is applied to excite simultaneously both energies $E_{n^{\prime}=0}$ and $E_{n=9}$. In this way, a new superposition $\Phi(t)$ (see eq 2) of the overlapping resonances is created, where similarly as in the single-pulse superpositions $\xi_{E_{n^{\prime}=0}}(t)$ and $\xi_{E_{n=9}}(t)$, the dominant contributions are those of the $n^{\prime}=0, n=8,9$, and 11 resonances. Thus, the $\Phi(t), \xi_{E_{n^{\prime}=0}}(t)$, and $\xi_{E_{n=9}}(t)$ superpositions have a qualitatively similar composition of relative amplitudes of the contributing overlapping resonances, but with the difference in the case of $\Phi(t)$ that the resonances can interfere, and the interference mechanism can be optimized to enhance the superposition lifetime. Such optimization is achieved by varying the delay time between the two pulses of $\mathcal{E}_{\text {pump }}(t)$.

The $\mathcal{E}_{\text {pump }}(t)$ field of eq 1 has been applied with different delay times $\Delta t$ between the two pulses exciting the $E_{n^{\prime}=0}$ and $E_{n=9}$ energies. The $I(t)$ survival probabilities of the superpositions obtained for three of these $\Delta t$ values are displayed in Figure 2. The curves display pronounced undulations caused by the interference between the resonances overlapping at the two energies excited. In order to obtain the lifetime associated with each superposition created, the corresponding $I(t)$ curve is fitted by using eq 4 . The fits associated with the three $I(t)$ curves of Figure 2 are also shown in the figure. It is found that eq. 4 fits very nicely the different $I(t)$ curves, thus providing reliable lifetimes through the range of $\Delta t$ investigated.

As previously discussed, ${ }^{21}$ the mechanism of quantum interference between the overlapping resonances requires simultaneous excitation of amplitude to the two energies used to induce interference. This requirement implies temporal overlap, at least partially, of the two pulses of $\mathcal{E}_{\text {pump }}(t)$, which means that the delay time between them must be smaller than the sum of their half temporal widths. Thus, the temporal width of the pulses applied determines the range of $\Delta t$ in which interference between the overlapping resonances takes place. For the present case where the width of the pulses is $\mathrm{FWHM}=200 \mathrm{ps}$, the corresponding range of $\Delta t$ leading to interference is $-300 \mathrm{ps} \leq \Delta t \leq 300 \mathrm{ps}$, which is the range investigated in this work. It is noted, however, that for $|\Delta t|>200$ ps temporal overlap of the pulses decreases 
rapidly, which reduces correspondingly the temporal region and the intensity of interference in $I(t)$. As a result, for $|\Delta t|=300$ ps the effect of interference on $I(t)$, albeit still present, is already rather small, and the shape of $I(t)$ approaches very much the shape of the sum of two separate, independent survival probablities of the single-pulse superpositions $\xi_{E_{n^{\prime}=0}}(t)$ and $\xi_{E_{n=9}}(t)$. In this situation the lifetimes estimated for $|\Delta t|=300$ ps are less representative of the $\Phi(t)$ superposition as those obtained for $|\Delta t|<300$.

The lifetime of the $\Phi(t)$ superposition has been calculated for different $\Delta t$ values in the range $-300 \mathrm{ps} \leq \Delta t \leq 300 \mathrm{ps}$, and they are collected in Table 1 and plotted in Figure 3. The lifetime of $\Phi(t)$ shows a pronounced enhancement when $|\Delta t|$ decreases from $|\Delta t|=300$, reaching a maximum of $\tau=65$ ps at $\Delta t=120$ ps. This is a large lifetime enhancement with respect to the lifetimes of $\sim 4$ and $\sim 15$ ps of the two single-pulse superpositions $\xi_{E_{n^{\prime}=0}}(t)$ and $\xi_{E_{n=9}}(t)$, respectively. Thus, inducing interference between the overlapping resonances of the superposition created, and optimizing the interference mechanism by varying the delay time between the pulses used to induce the interference, leads to a much longer survival lifetime of the superposition.

The results of Table 1 and Figure 3 present several features that are worth discussing. The shape of the lifetime curve of Figure 3 is very similar to the shapes of the corresponding lifetime curves previously found ${ }^{18}$ for the survival probabilities of the individual $\psi_{n^{\prime}=0}$ and $\psi_{n=9}$ resonances within the $\Phi(t)$ superposition, $\mid<$ $\psi_{n^{\prime}=0}(t)|\Phi(t)>|^{2}$ and $\left|<\psi_{n=9}(t)\right| \Phi(t)>\left.\right|^{2}$, featuring the maximum value at $\Delta t=$ 120 ps in all cases. This result should not be very surprising, because it just indicates that the qualitative behavior of the superposition lifetime with $\Delta t$ is the same as that of the resonances that contribute dominantly to the superposition. But it has the interesting implication that it is possible to build a superposition of overlapping resonances with a behavior easy to predict, based on the previous knowledge of the behavior of those resonances contributing dominantly to the superposition. In this sense, it is worth noting that recently ${ }^{18}$ an empirical law was reported that allows 
one to predict the optimal delay time $\Delta t_{\max }$ for maximum lifetime enhancement of individual overlapping resonances, when two different energies involving those resonances are excited by a field like $\mathcal{E}_{\text {pump }}(t)$ of eq 1 preparing a superposition $\Phi(t)$. Such a law is

$$
\Delta t_{\text {max }}=a\left(F W H M / 2+\tau_{1}^{i s o}+\tau_{2}^{i s o}\right)
$$

where $a= \pm 1, F W H M / 2$ is the half temporal width of the pulses of $\mathcal{E}_{\text {pump }}(t)$ (i.e., $100 \mathrm{ps}$ in the current simulations), and $\tau_{1}^{i s o}$ and $\tau_{2}^{i s o}$ are the lifetimes associated with the survival probability of the dominant ovelapping resonance at each of the two energies excited by $\mathcal{E}_{\text {pump }}(t)$ within the single-pulse superpositions (i.e., $I_{n^{\prime}=0}(t)=$ $\left|<\psi_{n^{\prime}=0}(t)\right| \xi_{E_{n^{\prime}=0}}(t)>\left.\right|^{2}$ and $I_{n=9}(t)=\left|<\psi_{n=9}(t)\right| \xi_{E_{n=9}}(t)>\left.\right|^{2}$ in the present case). For the cases of both the $n^{\prime}=0$ and $n=9$ resonances when FWHM=200 ps, the above law predicted $\Delta t_{\max }=120 \mathrm{ps}$, the same result as that of Figure 3. This seems to indicate that the same law of eq 5 can be applied to predict the value of $\Delta t_{\max }$ for a superposition $\Phi(t)$ of the form of eq 2. Confirmation of this result would require additional simulations in the line of those reported in ref 18 , which are out of the scope of the present work.

It is now interesting to analyze how the mechanism of interference constributes to enhance the lifetime of the resonance superposition. To this purpose, the behavior of the survival probabilities $I_{k}(t)=\left|<\psi_{k}(t)\right| \Phi(t)>\left.\right|^{2}\left(k=n^{\prime}, n\right)$ of the different overlapping resonances excited in the superposition $\Phi(t)$ is analized. It is noted that in the case of overlapping resonances, the sum of all the $I_{k}(t)=\mid\left\langle\psi_{k}(t)|\Phi(t)>|^{2}\right.$ $\left(k=n^{\prime}, n\right)$ survival probabilities does not completely match the expression of $I(t)=$ $|<\Phi(t)| \Phi(t)>\mid$, as can be easily checked with simple algebra. This match does indeed occur for nonoverlapping, orthogonal resonances. The reason of the difference between the two expressions is that a few interference terms do not coincide. It has been checked, however, that the difference is numerically very small, indicating that the different interference terms in the two expressions are rather negligible. In Figures $4 \mathrm{a}$ and $5 \mathrm{a}$ the $I_{k}(t)$ curves are displayed along with the survival probability of the $\Phi(t)$ superposition created at two different delay times, $\Delta t=-100$ and 120 
ps, respectively. In addition to the $I_{k}(t)$ curves, it is also very convenient to use in the analysis the normalized survival probabilities, defined as

$$
I_{k}^{\text {norm }}(t)=\frac{I_{k}(t)}{\sum_{i} I_{i}(t)}
$$

The $I_{k}^{n o r m}(t)$ curves are displayed in Figures $4 \mathrm{~b}$ and $5 \mathrm{~b}$ for the different resonances.

Figures 4 and 5 show that the main resonances contributing to the superposition prepared are $n^{\prime}=0, n=8,9$, and 11 , as previously discussed. All the $I_{k}(t)$ curves of Figures 4a and 5a display pronounced undulations due to interference between the overlapping resonances. Such undulations are separated by the same amount of time, $\sim 24 \mathrm{ps}$, in all the curves of both figures. This time separation between the peaks of the curves is the inverse of the energy difference $E_{n^{\prime}=0}-E_{n=9}$.

While all the $I_{k}(t)$ curves of Figures $4 \mathrm{a}$ and $5 \mathrm{a}$ display the same time separation between their peaks, they are not in phase, that is, the maxima and minima of the different $I_{k}(t)$ do not coincide. The dephasing of the survival probabilities is due to the combination of two factors. The main factor is that the two pulses of $\mathcal{E}_{\text {pump }}(t)$ excite the same overlapping resonances, but with different amplitude at each energy $E_{n^{\prime}=0}$ or $E_{n=9}$ (see Figure 1). Thus, the total contribution of a given resonance to the superposition at a gven time will depend on the specific $\Delta t$ value, and this will affect its mechanism of interference with the other resonances. The other factor is that each resonance has associated a different decay lifetime, which determines the decay of its amplitude and therefore also affects its mechanism of interference. It is noted, however, that despite the dephasing of the $I_{k}(t)$ curves, the superposition survival probability still retains the same separation of $\sim 24$ ps between the peaks.

The dephasing of the different $I_{k}(t)$ curves is closely related to the mechanism of interference between the resonances, and thus is likely to be the key aspect of the enhancement of the superposition lifetime. As discussed above, it has an intrinsic origin (the decay lifetime of each resonance) and a controllable origin (the delay time $\Delta t$ between the pulses). The $I_{k}^{n o r m}(t)$ curves of Figures $4 \mathrm{~b}$ and $5 \mathrm{~b}$ reflect more clearly than the $I_{k}(t)$ ones the depahasing between them. Thus, in order to analyze in more detail the relation of the dephasing to the enhancement of the superposition 
lifetime, let us focus on the $I_{k}^{\text {norm }}(t)$ curves, and more specifically on those of the $n^{\prime}=0, n=8,9$, and 11 dominating resonances. It is stressed that the values of the $I_{k}^{n o r m}(t)$ curves should be taken with caution for those times when the resonances (and then the corresponding superposition) have decayed completely.

Both in Figure $4 \mathrm{~b}$ and $5 \mathrm{~b}$ it is found that $I_{n^{\prime}=0}^{\text {norm }}(t)$ and $I_{n=8}^{\text {norm }}(t)$ are nearly in phase most of the time, while they are both in opposite phase with $I_{n=9}^{\text {norm }}(t)$. The $I_{n=11}^{\text {norm }}(t)$ curve shows an intermediate phase situation as compared with the other three curves (not in phase, nor with completely opposite phase). This behavior is very similar for both delay times $\Delta t=-100$ and $120 \mathrm{ps}$, with the main difference between Figure $4 \mathrm{~b}$ and $5 \mathrm{~b}$ being the time at which the maximum intensity of interference takes place, which is determined by $\Delta t$. A similar behavior is also found for other $\Delta t$ values. Such a behavior indicates that the amplitude initially excited to the different resonances by $\mathcal{E}_{\text {pump }}(t)$ is transferred to other resonances back and forth along time. This transfer of amplitude occurs in phase for some resonances, which in turn are in opposite phase with other resonances, and are in an intermediate phase situation with a third group of resonances. In principle the transfer of amplitude takes place among all the resonances of the superposition during the time they are interfering, although the dominant ones are those determining the global behavior of the superposition.

The transfer of amplitude between different resonances back and forth has the effect of increasing the effective lifetime of the different individual resonances, and therefore the global lifetime of the superposition. In the absence of interference, in the case of a single-pulse superposition, each resonance decays according to its lifetime: The short-lived resonances decay fast and the long-lived ones take longer. When interference takes place, the transfer of amplitude between resonances back and forth makes possible a new decay process. Amplitude initially excited to shortlived resonances migrates to longer-lived ones, and after some time all or only part of this amplitude will come back to the short-lived resonances. This new process of amplitude transfer back and forth replaces the process of intrinsic resonance 
decay without interference. The interference terms contributing to $I(t)$ (as well as to the $I_{k}^{n o r m}(t)$ curves of Figures 4 and 5) play a crucial role in the new decay process, since they strongly modulate the shape of $I(t)$ as the exchange of amplitude between resonances takes place. As a result, the interference induced process has a longer duration than the intrinsic decay process, and it is what determines the enhancement of the superposition lifetime. In other words, opening the possibility for the amplitude to explore the different resonances of the superposition through interference, makes it more resistant to decay and increases its lifetime.

The existence of an optimal delay time between pulses leading to maximum enhancement of the superposition lifetime $(\Delta t=120 \mathrm{ps}$ in the present case) is related to the optimization of the mechanism of interference between the resonances. More specifically, this optimization is based on the maximization of the intensity of interference between resonances. Indeed, as the intensity of resonance interference and threfore of the interference terms is maximized, the duration of the process of amplitude transfer between resonances back and forth will be correspondingly maximized, optimizing the enhancement of the superposition lifetime. The conditions of this optimization have been qualitatively discussed earlier, ${ }^{18}$ and the law of eq 5 allows one to make a quantitative prediction of the corresponding $\Delta t_{\max }$.

\section{Conclusions}

The performance of a weak-field coherent control scheme aimed at delaying the decay of a superposition state composed of overlapping resonances is investigated.

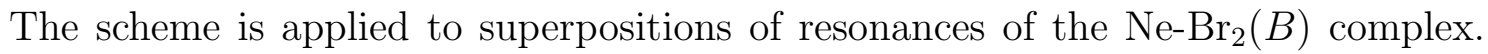
The control scheme uses a pump laser field consisting of two pulses delayed in time, each pulse exciting a different energy of the excitation spectrum of the Ne$\operatorname{Br}_{2}\left(B, v^{\prime}=35\right)$ ground resonance state at which several overlapping resonances are simultaneously populated. In this way interference between the overlapping resonances is induced, and the lifetime of the superposition created is enhanced. By varying the delay time between the pulses the interference mechanism betwen reso- 
nances can be optimized, leading to maximize the lifetime enhancement. Maximum enhancement of the superposition lifetime is achieved for a delay time between the pulses of 120 ps. This optimal delay time is the same as that previously found for maximum lifetime enhancement for the two individual resonances that dominate the present superposition. Not surprisingly, this result indicates that the behavior of the superposition with respect to optimization of lifetime control is the same as that of the dominant individual resonances. Thus, the same law previously reported to predict the optimal delay time for the individual resonances can be applied to the superposition state.

Analysis of the effect of the interference mechanism on the lifetime enhancement reveals that interference induces a process of transfer of amplitude back and forth between the resonances, that replaces the intrinsic decay proces occurring in the absence of interference. The amplitude transfer process has a longer duration than the intrinsic decay, making the superposition state a longer-lived one. The advantage of the present control scheme is twofold. On the one hand, it is simple to apply because optimization of the field requires only to fit the delay time betwen the pulses, and not to optimize the field shape. On the other hand, the behavior of the superposition regarding the control optimization is easily predictable by applying a law previously suggested for individual resonances.

\section{AUTHOR INFORMATION}

\section{Corresponding author}

E-mail: garciavela@iff.csic.es

\section{ORCID}

0000-0002-1214-2132

\section{Notes}


The author declares no competing financial interests.

\section{ACKNOWLEDGMENTS}

This work was funded by the Ministerio de Economía y Competitividad (MINECO, Spain), Grant No. CTQ2015-65033-P. The Centro de Supercomputación de Galicia (CESGA, Spain) is acknowledged for the use of its resources.

\section{REFERENCES}

(1) Joos, E.; Zeh, H.D.; Kiefer, C.; Guilini, D.; Kupsch, J.; Stamatescu, L.O. Decoherence and the Appearance of a Classical World in Quantum Theory; Springer: Berlin, 2003.

(2) Schlosshauer, D. Decoherence and the Quantum-to-Classical Transition; Springer: Berlin, 2007.

(3) Diestler, D.J.; Zewail, A.H. Vibronic Depahsing of Anharmonic Molecules. I. Theory and Its Application to the Separability of Intra- and Intermolecular Processes. J. Chem. Phys. 1979, 71, 3103-3112.

(4) Nielsen, M.A.; Chuang, I.L. Quantum Computation and Quantum Information; Cambridge University Press: Cambridge, 2000.

(5) Lidar, D.A.; Chuang, I.L.; Whaley, K.B. Decoherence-Free Subspaces for Quantum Computation. Phys. Rev. Lett. 1998, 81, 2594-2597.

(6) Viola, L.; Knill, E.; Lloyd, S. Dynamical Decoupling of Open Quantum Systems. Phys. Rev. Lett. 1999, 82, 2417-2421.

(7) Vitali, D.; Tombesi, P. Using Parity Kicks for Decoherence Control. Phys. Rev. A 1999, 59, 4178-4186.

(8) Shapiro, E.A.; Walmsley, I.A.; Ivanov, M.Y. Suppression of Decoherence in a Wave Packet via Nonlinear Resonance. Phys. Rev. Lett. 2007, 98, 050501-1-4.

(9) Katz, G.; Ratner, M.A.; Kosloff, R. Decoherence Control by Tracking a Hamiltonian Reference Molecule. Phys. Rev. Lett. 2007, 98, 203006-1-4.

(10) Branderhorst, M.P.A.; Londero, P.; Wasytcyk, P.; Brif, C.; Kosut, R.L.; 
Rabitz, H.; Walmsley, I.A. Coherent Control of Decoherence. Science 2008, 320, 638-643.

(11) Biswas, A.; Shapiro, M.; Brumer, P. Overlapping Resonances in the Resistance of Superposition States to Decoherence. J. Chem. Phys. 2010, 133, 014103$1-7$.

(12) Gerbasi, D; Sanz, A.S.; Christopher, P.S.; Shapiro, M; Brumer, P. Overlapping Resonances in the Control of Intramolecular Vibrational Redistribution. $J$. Chem. Phys. 2007, 126, 124307-1-9.

(13) Rice, S.A.; Zhao, M. Optical Control of Molecular Dynamics; Wiley: New York, 2000.

(14) Christopher, P.S.; Shapiro, M; Brumer, P. Overlapping Resonances in the Coherent Control of Radiationless Transitions: Internal Conversion in Pyrazine. J. Chem. Phys. 2005, 123, 064313-1-9.

(15) Christopher, P.S.; Shapiro, M; Brumer, P. Efficient Partitioning Technique for Computing the Dynamics of Intramolecular Processes: Radiationless Transitions in Pyrazine. J. Chem. Phys. 2006, 124, 184107-1-9.

(16) García-Vela, A. Active Control of the Lifetime of Excited Resonance States by Means of Laser Pulses. J. Chem. Phys. 2012, 136, 134304-1-8.

(17) García-Vela, A. Strong Enhancement of the Lifetime of a Resonance State by Using a Combination of Two Laser Pulses. J. Phys. Chem. Lett. 2012, 3, 1941-1945.

(18) García-Vela, A. Unravelling the Mechanisms of Interference Between Overlapping Resonances. Phys. Chem. Chem. Phys. 2018, 20, 3882-3887.

(19) García-Vela, A.; Henriksen, N.E. Coherent Control of Photofragment Distributions Using Laser Phase Modulation in the Weak-Field Limit. J. Phys. Chem. Lett. 2015, 6, 824-829.

(20) García-Vela, A. Weak-Field Coherent Control of Molecular Photofragment State Distributions. Phys. Rev. Lett. 2018, 121, 153204-1-6.

(21) García-Vela, A. A Unified Theory of Weak-Field Coherent Control of the 
Behavior of a Resonance State. Phys. Chem. Chem. Phys. 2019, 21, 7491-7501.

(22) Juanes-Marcos, J.C.; García-Vela, A. An Energy-Resolved Study of the Partial Fragmentation Dynamics of Ar-HCl into $\mathrm{H}+\mathrm{Ar}-\mathrm{Cl}$ after Ultraviolet Photodissociation. J. Chem. Phys. 2000, 112, 4983-4993.

(23) García-Vela, A.; Villarreal, P.; Delgado-Barrio, G. An Approximate Quantal Treatment to Obtain the Energy Levels of Tetraatomic $\mathrm{X}-\mathrm{I}_{2}-\mathrm{Y}$ van der Waals Clusters (X,Y=He, Ne). J. Chem. Phys. 1990, 92, 497-507.

(24) Cabrera, J.A.; Bieler, C.R.; Olbricht, B.C.; van der Veer, W.E.; Janda, K.C. Time-Dependent Pump-Probe Spectra of $\mathrm{NeBr}_{2}$. J. Chem. Phys. 2005, 123, 054311-1-8

(25) Taylor, M.A.; Pio, J.M.; van der Veer, W.E.; Janda, K.C. Competition between Electronic and Vibrational Predissociation Dynamics of the $\mathrm{HeBr}_{2}$ and $\mathrm{NeBr}_{2}$ van der Waals Molecules. J. Chem. Phys. 2010, 132, 104309-1-8.

(26) García-Vela, A.; Janda, K.C. Quantum Dynamics of Ne-Br 2 Vibrational Predissociation: The Role of Continuum Resonances as Doorway States. J. Chem. Phys. 2006, 124, 034305-1-13.

(27) García-Vela, A. Highly Delocalized Orbiting Resonances. J. Chem. Phys. 2008, 129, 094307-1-5.

(28) García-Vela, A. Quantum Interference Control of an Isolated Resonance Lifetime in the Weak-Field Limit. Phys. Chem. Chem. Phys. 2015, 17, 2907229078 .

(29) García-Vela, A. Intramolecular Vibrational Redistribution in $\mathrm{Ne}^{-\mathrm{Br}_{2}}$ : The Signature of Intermediate Resonances in the Excitation Spectrum. J. Chem. Phys. 2007, 126, 124306-1-10.

(30) García-Vela, A. The Structure of a Resonance State. Chem. Sci. 2017, 8, $4804-4810$ 


\section{TABLE CAPTIONS}

Table 1. Calculated lifetimes of the resonance superposition prepared with $\mathcal{E}_{\text {pump }}(t)$ versus the delay time between the two exciting pulses of the field.

\section{FIGURE CAPTIONS}

Figure 1. Calculated excitation spectrum associated with the ground vdW resonance of $\mathrm{Ne}-\mathrm{Br}_{2}\left(B, v^{\prime}=35\right)$. The energy axis is relative to the $\mathrm{Ne}+\mathrm{Br}_{2}\left(B, v^{\prime}=35, j^{\prime}=\right.$ $0)$ dissociation threshold. The main peak of the spectrum (labeled as $\left(v^{\prime}, g r\right)$ and located at $-56.34 \mathrm{~cm}^{-1}$ ) corresponds mainly to the ground resonance of the $v^{\prime}=35$ vibrational manifold. The positions of identified resonances associated with the $v^{\prime}-1$ manifold in the range $n=5-13$ are indicated by arrows in the figure, at the energies (relative to the $\operatorname{Br}_{2}\left(B, v^{\prime}=35, j^{\prime}=0\right.$ ) + Ne dissociation threshold) -64.22 (for $n=5$ ), $-62.80,-60.76,-59.57$, $-57.75,-54.79,-54.23,-51.36$, and $-50.99 \mathrm{~cm}^{-1}$ (for $n=13$ ), respectively.

Figure 2. Survival probability $I(t)$ of the superposition created by applying the laser field $\mathcal{E}_{\text {pump }}(t)$ with three different delay times between the two pulses, $\Delta t=-100$, 80, and 110 ps. The plain, nonoscillating curve is a fit to $I(t)$ obtained using eq 4 in order to extract the corresponding lifetime.

Figure 3. Plot of the lifetimes of the resonance superposition collected in Table 1.

Figure 4. (a) Survival probabilities $I_{k}(t)=\left|<\psi_{k}(t)\right| \Phi(t)>\left.\right|^{2}\left(k=n^{\prime}, n\right)$ of the different overlapping resonances excited in the superposition $\Phi(t)$, along with the survival probability of the superposition itself for the delay time $\Delta t=-100$ ps. (b) Normalized survival probabilities of the overlapping resonances for $\Delta t=-100$ ps. See the text for details.

Figure 5. Same as Figure 4 but for $\Delta t=120$ ps. Note that the timescale here is somewhat longer than in Figure 4. 
TABLE 1

\begin{tabular}{cc}
\hline \hline$\Delta t(p s)$ & $\tau(p s)$ \\
\hline-300 & 4.0 \\
-100 & 6.5 \\
0 & 11.0 \\
80 & 34.0 \\
100 & 46.0 \\
110 & 52.0 \\
120 & 65.0 \\
140 & 48.0 \\
200 & 15.0 \\
300 & 6.0 \\
\hline \hline
\end{tabular}

